\title{
Grip strength following total wrist arthrodesis using the same hand as reference: a prospective study
}

\author{
Koch $\mathrm{O}^{1}{ }^{-}$, Alexander $\mathrm{AN}^{2}$, Olorunju $\mathrm{S}^{3}{ }^{\circledR}$, McLoughlin $\mathrm{HA}^{4}$, Le Roux $\mathrm{TL}^{5}$ \\ 1 MBChB(UP), MMed(Orth)UP, FC Orth SA; 1 Military Hospital, Department of Orthopaedic Surgery, University of Pretoria; Pretoria East Hospital \\ Suite M33 \\ $2 \mathrm{MBChB(UP)}$, FC Orth SA; Kalafong Hospital, Department of Orthopaedic Surgery, University of Pretoria \\ $3 \mathrm{PhD}$; Biostatistics Unit, South African Medical Research Council \\ 4 D Litt et Phil; Pretoria East Hospital Suite M33 \\ 5 MBChB(UP), MMed(Orth)UP FCS Ort SA; 1 Military Hospital, Department of Orthopaedic Surgery, University of Pretoria; Pretoria East Hospital \\ Suite M33
}

Corresponding author: Dr Odette Koch, Department of Orthopaedic Surgery, 1 Military Hospital, Hospital Road, Pretoria, 0187, South Africa; 289 Thatchers Fields, Lynnwood, 0081; cell: 0723145566; tel: 0129984203; email: okoch@okoch.co.za

\begin{abstract}
Introduction: Many patients suffer from a decrease in grip strength due to various conditions of the wrist, including osteoarthritis. A stable painless wrist is essential for normal function of the hand. Many patients are offered a total wrist fusion to alleviate their pain and increase their grip. There is a dearth of literature investigating the effect of total wrist arthrodesis on grip strength utilising the same side as a reference.

Methods: A prospective study was done to determine change in grip strength and patient-reported outcome measurements postoperatively as compared to same-side pre-operative values in subjects undergoing total wrist arthrodesis with a minimum follow-up of one year. Grip strength was measured pre-operatively and at least one year post-operatively using the Jamar® (Patterson Medical) dynamometer in the standardised method as part of set protocol. Functional outcomes were assessed by the patients completing Disabilities of Arm, Shoulder and Hand (DASH) scores, pre-operatively and at one-year follow-up. The differences in measures were assessed using a paired samples T-test, as well as percentage changes between measurements.

Results: Twenty-two patients were evaluated, with a mean age of 49.6 years (range 19-85). The mean follow-up was 27 months (12-52 months). The grip strength in the affected hand improved on average by $12.6 \mathrm{~kg}(\mathrm{p}<0.001 ; \mathrm{Cl} 95 \%$ [7.7, 17.3]). The grip in the affected hand was $84.5 \%$ compared to the pre-operative value of the unaffected side $(32.8 \mathrm{~kg} \mathrm{vs} 38.8 \mathrm{~kg} ; \mathrm{p}<0.001)$. The DASH score improved by 27.8 points $(p=0.1)$.

Conclusion: Total wrist fusion remains a reliable procedure to restore a power grip at the expense of wrist movement. It is a suitable option for a high-demand patient with an isolated wrist problem.
\end{abstract}

Level of evidence: Level 3

Keywords: wrist, fusion, grip strength, arthritis

Citation: Koch O, Alexander AN, Olorunju S, McLoughlin HA, Le Roux TL. Grip strength following total wrist arthrodesis using the same hand as reference: a prospective study. SA Orthop J 2019;18(4):41-45. http://dx.doi.org/10.17159/2309-8309/2019/v18n4a5

Editor: Dr A Ikram, Stellenbosch University, Cape Town, South Africa

Received: February 2019

Accepted: May 2019

Published: November 2019

Copyright: $\odot 2019 \mathrm{Koch}$ O, et al. This is an open-access article distributed under the terms of the Creative Commons Attribution Licence, which permits unrestricted use, distribution and reproduction in any medium, provided the original author and source are credited.

Funding: The authors confirm that no benefits of any form have been received from any party related directly or indirectly to the subject of this article.

Conflict of interest: The authors, their immediate family, and any research foundation with which they are affiliated did not receive any financial payments or other benefits from any commercial entity related to the subject of the article. 


\section{Introduction}

A stable painless wrist is essential for normal function of the hand. Many patients suffer from a decrease in grip strength due to various conditions of the wrist, including osteoarthritis. Highdemand patients are often offered a total wrist fusion as a possible solution to their pain and grip strength deficits.

Various studies have compared post-operative grip strength as a value of the contralateral side or as a comparison to the normative values for age and sex. ${ }^{1}$

A limited number of studies have quantified the gain in grip strength of the affected hand before surgery and after successful fusion. Rauhaniemi et al. ${ }^{2}$ published research where post-operative grip strength was measured as a percentage of the pre-operative value in 115 patients with rheumatoid arthritis following a total wrist arthrodesis. This study used the Mannerfelt technique. ${ }^{3}$ Right-hand grip strength was $24 \%$ stronger after one year, and $20 \%$ stronger if surgery was on the left hand. This is the most extensive series reported in the literature. A study similar to the current paper retrospectively reviewed the gain in grip strength in 39 cases and reported the pre-operative grip in the affected hand as $21 \mathrm{~kg}$ on average and $31 \mathrm{~kg}$ post-operatively (Meads et al. ${ }^{4}$ ). An AO plate and iliac crest autograft were used in this series by Meads.

The study objectives were to determine:

1. The gain in grip strength in the same hand as a percentage of the value pre-operatively

2. Grip strength after the successful fusion of the wrist as a percentage of the pre-operative value of the contralateral side

3. Grip strength as a percentage of the normative value (Mathiowetz ${ }^{1}$ )

4. The improvement in the Disability of Arm, Shoulder and Hand $(\mathrm{DASH})^{5}$ score from baseline values.

\section{Patients and methods}

A prospective observational study was performed at our institution. Twenty-five participants undergoing a total wrist fusion for osteoarthritic-related conditions from August 2013 to the current date were eligible for the study provided they could comply with a one-year minimum follow-up post-operatively. Twenty-two participants completed the study as required. One participant terminated employment and could not be reached for follow-up. Two participants voluntarily withdrew from the study before the final assessment.

Thirteen males and nine females underwent surgery. The mean age of the study population was 49.6 years (range 19-85). In 13 cases, the participant's dominant wrist was operated. The mean follow-up was 27 months (12-52 months).

Each participant completed the DASH questionnaire before surgery and at final follow-up. The participants did not have access to their personal pre-operative DASH form at follow-up. A Jamar dynamometer was used to measure the grip strength before surgery in all cases. All measurements were taken in a standardised position with the participant sitting with their arm held abducted next to the body and the elbow in $90^{\circ}$ flexion with the forearm in the neutral position. ${ }^{6}$ The Jamar was set on the second handle position for all participants. The average of three trials was used while allowing enough time for recovery in-between efforts to prevent fatigue. The dynamometer was calibrated according to manufacturer guidelines. ${ }^{7}$ The measurement was repeated at three-monthly intervals and finally at a minimum of 12 months after surgery.
Participants with known rheumatoid or seronegative arthritis, any neuromuscular disorders or tendon-related problems were excluded.

The surgery was performed by two surgeons (OK and TLB). The patient was positioned supine on the operating table with the arm positioned on a hand table. An above-elbow tourniquet was used in all cases. The skin incision was dorsal from the middle of the middle finger metacarpal and extended $5-7 \mathrm{~cm}$ proximal to the dorsal wrist crease. Through a dorsal approach between the third and fourth extensor compartments, the extensor pollicus longus muscle was identified and retracted radially. Transverse incisions were made at the proximal and distal end of the extensor retinaculum, and this structure was reflected ulnarly, underneath the tendons of the fourth compartment. The extensor retinaculum was reattached at the end of the procedure between the plate and the muscle to prevent tendon attrition. The distal part of the posterior interosseous nerve was resected, the Lister's tubercle was removed, and the cartilage on the articular surface between the radius and lunate, and lunate and capitate, was fishscaled. Though the third carpometacarpal joint was spanned, no particular attention was paid to the joint between the base of the middle finger and the capitate.

Bone graft was done in all cases, with an allograft bone graft used in 17 of the cases. In five of the cases, a partial or complete resection of the distal ulna was done in combination with the wrist fusion due to arthritis at the distal radioulnar joint. In these cases, the resected bone was used as an autograft. A Depuy Synthes ${ }^{\circledR}$ wrist arthrodesis plate was used in all cases. The plate was placed from the third metacarpal to the distal radius. The implant was used in compression mode for all procedures. With the plate in position, the wrist is in $10^{\circ}$ of wrist extension and positioned in neutral deviation.

Post-operative management included a volar splint for two weeks followed by a circular cast for another four weeks. Radiographs were repeated at the six-week follow-up visit. A hand therapist instructed the patients on the correct hand and finger movement while in the splint on the day of the surgery. The participants received a home programme on discharge and routinely attended hand therapy. The surgeons evaluated the participants' wrists at two and six weeks. An individualised follow-up regimen was followed up to one-year post-operatively.

All of the participants were followed up by the principal investigators. The participants included in the study can receive medical care only at our institution. The hospital provides healthcare service to members of the military and their dependents in KwaZulu-Natal, Mpumalanga, North West, Limpopo and Gauteng (provinces in South Africa). This accounts for a third of the country.

The referring units provide transport to and from the facility. All post-operative care and complications related to the wrist are treated at one hospital.

\section{Statistical analysis}

The data analyses were done in collaboration with the Biostatistics Unit of the Medical Research Council.

The following aspects of the analyses are of importance:

- The improvement in grip strength in the same hand as a percentage of the value pre-operatively, as a percentage of the contralateral side and as a percentage of the normative value

- The improvement in DASH scores

- Descriptive statistics (summary statistics, i.e. mean, variance)

- Confidence intervals $(\mathrm{Cl})$

- The paired t-test was used to compare the parameters of interest 
The test was applied between pre- and post-test scores for each of the parameters for individual patients, and served as his/her control. Furthermore, the change between pre- and post-test scores was tested using the one-sample t-test.

\section{Results}

Scaphoid non-union advanced collapse (SNAC) (5/22) and scapholunate advanced collapse (SLAC) (8/22) (Figure 1) accounted for $59 \%$ of the operative indications (Table I).

The grip strength in the affected hand improved on average by $12.6 \mathrm{~kg}$ ( $\mathrm{p}<0.001 ; \mathrm{Cl} 95 \%[7.7,17.3])$ from $20.2 \mathrm{~kg}(14.03,26.6)$ to $32.8 \mathrm{~kg}(27.2,38.32)$. The grip strength in the affected hand was $84.5 \%$ compared to the pre-operative value of the unaffected side ( $32.8 \mathrm{~kg}$ vs $38.8 \mathrm{~kg} ; \mathrm{p}<0.001)$. In comparison to the normative values as defined by Mathiowetz, ${ }^{1}$ the post-operative grip in the operated wrist was $85.4 \%(\mathrm{p}<0.001)$ (Table II).

The DASH score improved by 27.8 points from 52.0 preoperatively to 24.2 post-operatively, although this improvement was not statistically significant $(p=0.1)$.

Eleven participants had undergone previous surgery to the same wrist preceding the total wrist fusion. Five had associated ulnar-sided surgery at the same setting (three matched distal ulnar resections and two Darrach procedures).

Four participants required follow-up surgery. One participant was scheduled for carpal tunnel surgery and requested removal of the hardware during the same procedure (Table III). One patient developed a non-union and needed four subsequent operations before union was achieved. He initially had a high-velocity distal radius fracture on the non-dominant hand and had undergone multiple surgeries before the wrist fusion. This participant worked as a maintenance and recovery official at his unit and continued with his manual labour after successful fusion.

The current study populations' job requirements are unique because of the need to handle a rifle and do physical training. The permanent force military members all returned to work with duty restrictions within six weeks following the surgery. One participant changed his dominance. He was ambidextrous and continued to play action cricket at a provincial level after the wrist fusion. Three participants required reclassification with duty restrictions because they could not handle a rifle with the affected wrist and could not do push-ups. They were, however, able to handle a handheld weapon. One participant was reskilled, and one participant changed her occupation, but this was unrelated to the wrist fusion. All of the pensioners in the study went back to their hobbies but made adjustments. Specific actions like turning a doorknob and opening a tight jar were difficult.

Participants reported improvement of pain after the wrist fusion. Question number 24 of the DASH score was evaluated. Preoperatively, the participants scored their pain as follows: $21 \%$ extreme, $63 \%$ severe, $10 \%$ moderate and $5 \%$ mild. Post-operatively, the score improved to $21 \%$ moderate, $42 \%$ mild and $36 \%$ no pain.

The radiological union was not evaluated by a radiologist as the hospital does not routinely receive radiology reports. The X-rays were, however, assessed by the attending physician and correlated clinically with the appearance of the wrist, amount of pain, and swelling in the region of the implant (Figure 2).

\section{Discussion}

Reliable evaluation of grip strength is essential to evaluate the effectiveness of the different surgical options in the management of arthritic conditions of the wrist. ${ }^{8}$ Theoretically, loss of grip strength is a consequence of total wrist arthrodesis. The patients who suffer from arthritis in the wrist due to various reasons are in good health generally, but have an isolated problem in the wrist, which results in

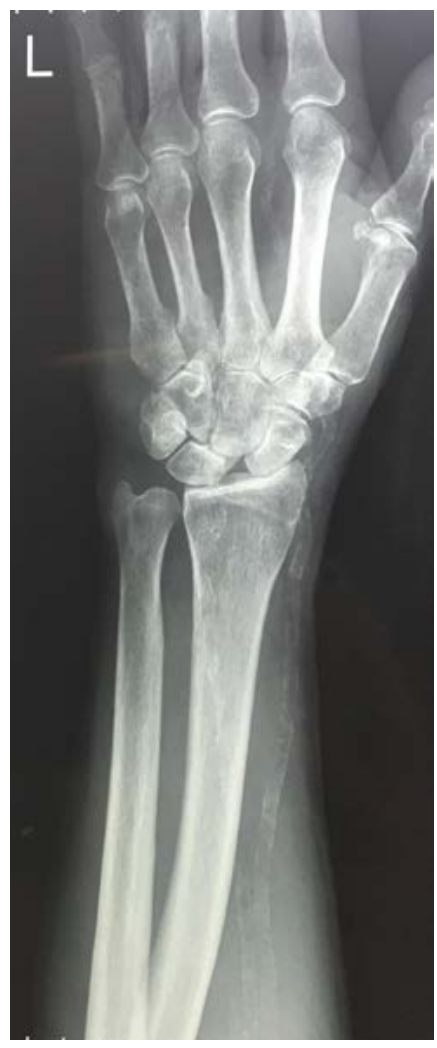

Figure 1. (left) Pre-operative radiograph of a SLAC wrist with age-related atherosclerosis

Figure 2. (below) Post-operative radiograph 12 months after wrist arthrodesis
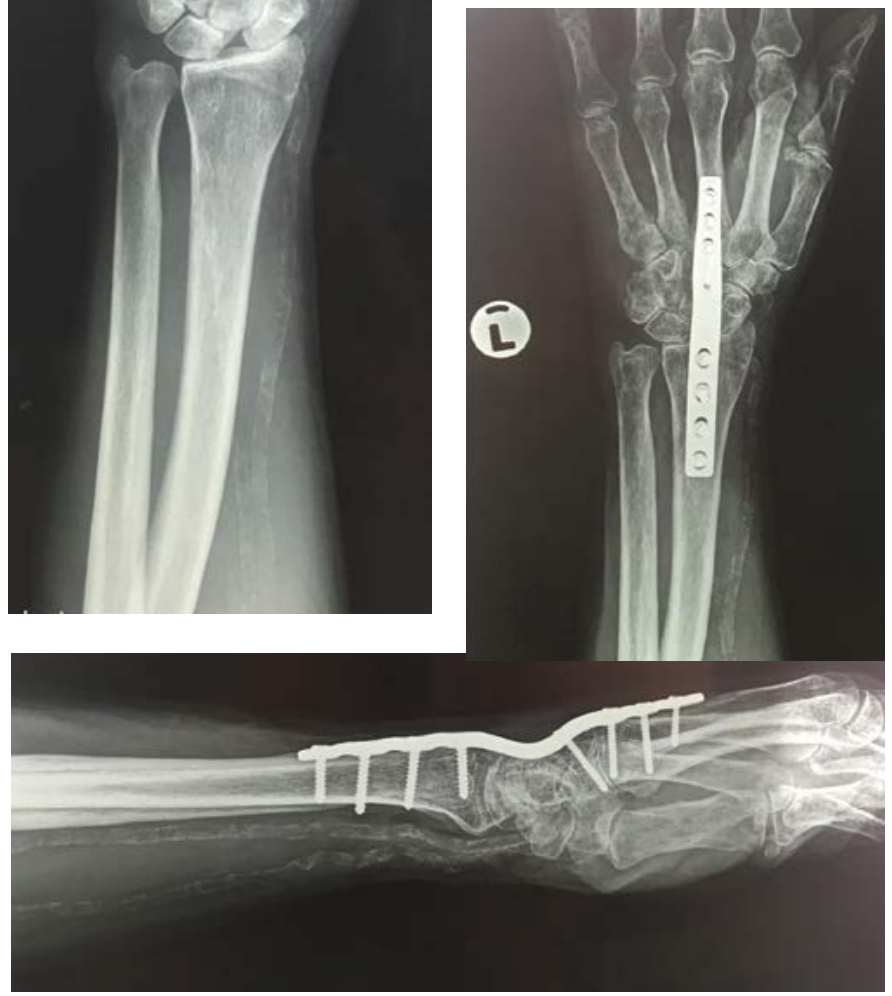

Table I: Indications for surgery

\begin{tabular}{|l|l|l|}
\hline \multicolumn{1}{|c|}{ Wrist condition } & \multicolumn{1}{c|}{ Total } & Percentage \\
\hline SNAC & 5 & 23 \\
\hline SLAC & 8 & 36 \\
\hline Primary osteoarthrosis & 5 & 23 \\
\hline Post-traumatic conditions & 3 & 14 \\
\hline $\begin{array}{l}\text { Secondary osteoarthrosis due to } \\
\text { sepsis }\end{array}$ & 1 & 4 \\
\hline Total & $\mathbf{2 2}$ & $\mathbf{1 0 0}$
\end{tabular}

SNAC: scaphoid non-union advanced collapse; SLAC: scapholunate advanced collapse

disability. Weak grip strength is already present in the affected wrist due to the underlying condition. Improvement in the power of the grip leads to improvement of this isolated impairment.

Various authors have found the loss of grip strength to range from $57 \%$ to $78 \%^{2,4,9-11}$ compared to the unaffected side. The current study examined the grip strength in the affected hand preoperatively as a value of the grip strength after successful fusion. In the population studied, the manual labourer runs the risk of losing their job if they are not able to comply with work requirements. These requirements often include a power grip. This study has shown that grip strength improved by $62.3 \%$ from the starting value and this is $84.5 \%$ of the value of the contralateral side. The significant gain in grip strength compared to the other authors might be attributed to the prolonged follow-up. 
Table II: Summary statistics

\begin{tabular}{|c|c|c|c|c|c|c|c|c|c|}
\hline Sex & $\begin{array}{l}\text { Summary } \\
\text { statistics }\end{array}$ & Age (yrs) & DASH pre & DASH post & Grip pre & Grip post & Contra & $\begin{array}{l}\text { Normative } \\
\text { affected }\end{array}$ & $\begin{array}{c}\text { Normative } \\
\text { contra }\end{array}$ \\
\hline \multirow[t]{5}{*}{ Female } & $\mathrm{n}=9$ & & & & & & & & \\
\hline & Mean & 54.8 & 60.7 & 31.1 & 12.9 & 21.9 & 26.7 & 24.9 & 25.4 \\
\hline & Min & 29.0 & 17.9 & 7.1 & 0.7 & 9.3 & 14.7 & 18.6 & 17.0 \\
\hline & Max & 85.0 & 88.3 & 63.8 & 23.7 & 31.3 & 43.7 & 35.7 & 32.0 \\
\hline & Median & 61.0 & 56.0 & 31.0 & 14.3 & 20.3 & 24.7 & 20.7 & 25.0 \\
\hline \multirow[t]{5}{*}{ Male } & $n=13$ & & & & & & & & \\
\hline & Mean & 46.1 & 46.0 & 19.5 & 25.3 & 40.3 & 47.2 & 47.8 & 45.8 \\
\hline & Min & 19.0 & 20.8 & 5.0 & 5.0 & 22.3 & 20.7 & 29.4 & 34.2 \\
\hline & Max & 70.0 & 67.5 & 55.8 & 56.7 & 62.3 & 78.0 & 55.2 & 54.9 \\
\hline & Median & 52.0 & 47.5 & 19.0 & 22.7 & 41.0 & 45.7 & 50.1 & 47.4 \\
\hline \multirow[t]{5}{*}{ Total } & $n=22$ & & & & & & & & \\
\hline & Mean & 49.6 & 52.0 & 24.2 & 20.2 & 32.8 & 38.8 & 38.4 & 37.5 \\
\hline & Min & 19.0 & 17.9 & 5.0 & 0.7 & 9.3 & 14.7 & 18.6 & 17.0 \\
\hline & Max & 85.0 & 88.3 & 63.8 & 56.7 & 62.3 & 78.0 & 55.2 & 54.9 \\
\hline & Median & 52.5 & 51.8 & 20.6 & 16.8 & 34.8 & 43.2 & 41.0 & 34.8 \\
\hline
\end{tabular}

Table III: Wrists requiring follow-up surgery

\begin{tabular}{l|c|}
\hline Non-union & 1 \\
\hline Carpal tunnel syndrome & 1 \\
\hline Removal of hardware & 2 \\
\hline $\begin{array}{l}\text { Persistent ulnar-sided wrist pain requiring ulnar-sided } \\
\text { surgery }\end{array}$ & 1 \\
\hline Total & $\mathbf{1 8 . 2} \%$
\end{tabular}

In a recent review article by Wei and Feldon ${ }^{12}$ major complications were described as wrists requiring follow-up surgery, and minor complications as those not requiring any follow-up surgery. In the 45 studies included, reporting on 1782 wrists, the major complication rate was $19 \%$ and the minor complication rate $10 \%$. The advances in implants have successfully decreased the complications reported in total wrist fusion surgery. A 1996 study reported a $51 \%$ complication rate with a high re-operation rate, mostly due to tendon-related issues..$^{13}$ The major complication rate in the current study was $18.2 \%$.

Adey et al. ${ }^{14}$ reported 14 out of 22 patients still experienced pain after successful fusion. They speculate the sources of pain to be implant-related or due to neuroma formation. The results of the current population are similar, with 13 participants reporting mild to moderate pain at final follow-up. A benefit of the procedure is subjective improvement of pain. ${ }^{15}$ Although the pain experienced at the last follow-up was less than initially, only seven participants reported complete alleviation of pain. Total pain relief after wrist fusion is infrequent, and sources of pain remain debatable.

The optimal position of the fused wrist remains controversial. Grip strength is optimal with the wrist in $45^{\circ}$ of extension ${ }^{16}$ and the inability to restore grip to normative value might be due to suboptimal wrist positioning. Extreme extension of the wrist in a fused position might cause median nerve-related problems, and is cosmetically less acceptable. Field et al. ${ }^{17}$ concluded that the most suitable position for the wrist remains uncertain. The $A O$ plate incorporates $10^{\circ}$ of extension and neutral deviation.

When a patient has a deficient grip and discomfort, it causes disability in upper limb function. A total wrist fusion is considered a salvage operation, with the prerequisite that it improve persistent wrist pain. ${ }^{17}$ Because of the limitations after a wrist fusion, the procedure is often postponed, while several other motionpreserving operations are offered. Meads ${ }^{4}$ states that a wrist fusion should be recommended earlier, limiting the number of operations before the wrist fusion, but the patient should be warned about the limitations after the fusion. We support Meads' opinion that a wrist fusion should be offered sooner, as 11 participants had a total of 23 procedures to the wrist preceding the fusion.

Although Barbieri et al. ${ }^{10}$ reported controversies regarding the patients' subjective perception of overall upper limb function after total wrist fusion, $65.7 \%$ of their study population with low-demand work requirements went back to the same occupation after the surgery. In a study reporting on bilateral wrist fusions, most patients adapted to their limited wrist motion and were satisfied with their functionality. ${ }^{18}$ In another study, Hastings et al. ${ }^{13}$ reported a $64.5 \%$ return to the same work activities performed pre-operatively. Three participants with ultra-high demand occupations needed permanent duty restrictions and one participant was retrained. The remainder of the participants returned to the same occupation or hobbies.

\section{Conclusion}

Total wrist fusion remains a reliable procedure to restore a power grip and provide pain relief at the expense of wrist movement. It is a suitable option for a high-demand patient with an isolated wrist problem and should remain in the skill set of the orthopaedic surgeon.

\section{Ethics statement}

This study was approved by 1 Military Hospital Research Ethics Committee adhering to Good Clinical Practice/International Conference on Harmonisation and South African Clinical Trial Guidelines. Ethics approval number: 1MH/302/6. Informed consent was obtained from all participants before surgery. The anonymous datasheets and DASH questionnaires were numbered in sequential order to protect the participants' confidentiality.

\section{Declarations}

The authors declare authorship of this article and that they have followed sound scientific research practice. This research is original and does not transgress plagiarism policies. 


\section{Author contributions}

OK and TLR conceived of the research idea and were the primary surgeons in all cases. OK further assisted with application to the departmental research committee and ethics board; developed the study protocol and gathered patient data; contributed to writing up the manuscript for submission.

ANA developed the study protocol and gathered patient data.

SO contributed to writing up the manuscript for submission.

HAM assisted with application to the departmental research committee and ethics board; contributed to writing up the manuscript for submission.

\section{ORCID}

O Koch (D) https://orcid.org/0000-0003-1871-9569

S Olorunju (D) https://orcid.org/0000-0002-5598-8090

\section{References}

1. Mathiowetz V, Kashman N, Volland G, Weber K, Dowe M, Rogers S. Grip and pinch strength: normative data for adults. Arch Phys Med Rehabil 1985;66(2):69-74. doi:https://www.ncbi.nlm.nih.gov/ pubmed/3970660

2. Rauhaniemi J, Tiusanen $\mathrm{H}$, Sipola E. Total wrist fusion: a study of 115 patients. The Journal of Hand Surgery: British \& European Volume 2005;30(2):217-19. doi:10.1016/j.jhsb.2004.11.008

3. Mannerfelt L, Malmsten M. Arthrodesis of the wrist in rheumatoid arthritis. A technique without external fixation. Scand J Plast Reconstr Surg 1971;5(2):124-30. doi:10.3109/02844317109042952

4. Meads BM, Scougall PJ, Hargreaves IC. Wrist arthrodesis using a Synthes wrist fusion plate. The Journal of Hand Surgery: British \& European Volume 2003;28(6):571-74. doi:10.1016/ S0266-7681(03)00146-3

5. Hudak PL, Amadio PC, Bombardier C. Development of an upper extremity outcome measure: the DASH (disabilities of the arm, shoulder and hand) [corrected]. The Upper Extremity Collaborative Group (UECG). Am J Ind Med 1996;29(6):602-608. doi:10.1002/ (SICl)1097-0274(199606)29:6<602::AID-AJIM4>3.0.CO;2-L

6. Mathiowetz V, Weber K, Volland G, Kashman N. Reliability and validity of grip and pinch strength evaluations. The Journal of Hand Surgery 1984;9(2):222-26. doi:10.1016/S0363-5023(84)80146-X

7. Fess EE. A method for checking Jamar dynamometer calibration. Journal of Hand Therapy 1987;1(1):28-32. doi:10.1016/ S0894-1130(87)80009-1

8. Fong $\mathrm{PW}, \mathrm{Ng} \mathrm{GY}$. Effect of wrist positioning on the repeatability and strength of power grip. Am J Occup Ther 2001;55(2):212-16. doi:10.5014/ajot.55.2.212

9. De Smet L, Truyen J. Arthrodesis of the wrist for osteoarthritis: outcome with a minimum follow-up of 4 years. Journal of Hand Surgery (British and European Volume) 2003;28(6):575-77. doi:10.1016/S0266-7681(03)00208-0

10. Barbieri $\mathrm{CH}$, Mazzer N, Elui VMC, Fonseca MCR. Resultados funcionais da artrodese do punho (Functional results of wrist arthrodesis). Acta Ortopédica Brasileira 2002;10(1):17-24. doi:10.1590/S1413-78522002000100003

11. Leighton RK, Petrie D. Arthrodesis of the wrist. Canadian journal of surgery. Journal Canadien de Chirurgie 1987;30(2):115-16.

12. Wei $\mathrm{DH}$, Feldon $\mathrm{P}$. Total wrist arthrodesis indications and clinica outcomes. J Am Acad Orthop Surg 2017;25(1):3-11. doi:10.5435/ JAAOS-D-15-00424

13. Hastings $H$ 2nd, Weiss AP, Quenzer D, Wiedeman GP, Hanington $\mathrm{KR}$, Strickland JW. Arthrodesis of the wrist for post-traumatic disorders. The Journal of Bone and Joint Surgery. American Volume 1996;78(6):897-902. doi:10.1007/s00238-011-0556-3

14. Adey L, Ring D, Jupiter, Jesse B. Harvard Medical School, Boston, MA. Health status after total wrist arthrodesis for posttraumatic arthritis. J Hand Surg. 2005;30(5):932-36. doi:10.1016/j. jhsa.2005.06.004

15. Birch A, Nuttall D, Stanley JK, Trail IA. The outcome of wrist surgery: what factors are important and how should they be reported? The Journal of Hand Surgery, European Volume 2011;36(4):308-14. doi:10.1177/1753193410396647

16. Bhardwaj P, Nayak SS, Kiswar AM, Sabapathy SR. Effect of static wrist position on grip strength. Indian Journal of Plastic Surgery 2011;44(1):55-58. doi:10.4103/0970-0358.81440
17. Field $\mathrm{J}$, Herbert $\mathrm{TJ}$, Prosser $\mathrm{R}$. Total wrist fusion: A functional assessment. The Journal of Hand Surgery: British \& European Volume 1996;21(4):429-33. doi:10.1016/S0266-7681(96)80039-8

18. Wagner ER, Elhassan BT, Kakar, Sanjeev Department of Orthopedic Surgery, Division of Hand Surgery, Mayo Clinic, Rochester, MN. Long-term functional outcomes after bilateral total wrist arthrodesis. J Hand Surg 2015;40(2):224-28. doi:10.1016/j. jhsa.2014.10.032 